\title{
Emprendimiento sostenible: un estudio de caso múltiple
}

\author{
Julio C. Acosta-Prado ${ }^{1,2, *}$, Rodrigo A. Zárate-Torres ${ }^{3}$ y Edward A. Ortiz ${ }^{4}$ \\ (1) Facultad de Ciencias Empresariales, Universidad del Pacífico, 15072 Lima, Perú. \\ (2) Facultad de Ciencias Contables, Económicas y Administrativas, Universidad de Manizales, 170001 Manizales, \\ Colombia. \\ (3) Colegio de Estudios Superiores de Administración, 110311 Bogotá, Colombia. \\ (4) Facultad de Administración, Finanzas y Ciencias Económicas, Universidad EAN, 110221 Bogotá, Colombia. \\ (correo-e: jc.acostap@up.edu.pe; rodrigo.zarate@cesa.edu.co; eortiz_11@universidadean.edu.co)
}

Recibido May. 15, 2021; Aceptado Jul. 12, 2021; Versión final Ago. 4, 2021, Publicado Dic. 2021

\section{Resumen}

El principal objetivo del presente estudio es caracterizar el emprendimiento sostenible a partir de un diseño de caso múltiple. Con base al análisis de cinco casos, se describió el proceso de emprendimiento sostenible. Las respuestas de los entrevistados se analizaron a partir de un conjunto de pasos: 1) codificación de las respuestas, 2) categorización en temáticas comunes y 3) establecimiento de conclusiones que permitan una explicación general agrupada en los temas comunes. Los resultados identificados para el emprendimiento sostenible son: 1) el reconocimiento de una problemática social o ambiental, 2) el reconocimiento de una oportunidad social o ambiental, 3) el desarrollo de una solución de doble resultado, 4) el desarrollo de una solución de triple resultado, 5) la financiación y formación de una organización sostenible, y 6) la creación o ingreso a un mercado sostenible. Se concluye que estos hallazgos permiten realizar contribuciones teóricas y prácticas de emprendimiento sostenible.

Palabras clave: sostenibilidad; emprendimiento; triple resultado; caso múltiple

\section{Sustainable entrepreneurship: a multi-case study}

\begin{abstract}
The main objective of the present study is to characterize sustainable entrepreneurship by using a multiplecase design. Based on the analysis of five cases, the process of sustainable entrepreneurship is described. Interviewees' responses are analyzed following a set of steps: 1) response codification, 2) categorization into common themes, and 3) conclusions that provide a general explanation by grouping common themes. The results show that there is: 1) a recognition of a social or environmental problem, 2) a recognition of a social or environmental opportunity, 3) development of a double bottom line solution, 4) development of a triple bottom line solution, 5) financing and formation of a sustainable organization, and (6) creation or entry into a sustainable market. It is concluded that these findings allow making theoretical and practical contributions to sustainable entrepreneurship.
\end{abstract}




\section{INTRODUCCIÓN}

A finales del siglo pasado, el informe Brundtland de Comisión Mundial sobre el Medio Ambiente y el Desarrollo planteó la necesidad de crear una agenda global para el cambio y compartir las percepciones sobre los problemas medioambientales a largo plazo, la desigualdad social y los esfuerzos para afrontar los problemas de protección y mejora del medio ambiente, así como proteger los intereses de las próximas generaciones (WCED, 1987). El informe destaca cuestiones como el desarrollo social y económico de las empresas, surgiendo el concepto de desarrollo sostenible. Según esta idea, la sostenibilidad consiste en preservar la existencia humana, que depende del funcionamiento de los sistemas económicos, sociales y políticos, así como de un entorno físico y biológico favorable (Sulphey, 2019). De esta manera, el desarrollo global y los retos a los que se enfrenta la sociedad actual han generado una mayor concientización en las empresas y gobiernos hacia un enfoque más eficiente de desarrollo sostenible (Tsaurkubule, 2016).

El desarrollo sostenible busca satisfacer las necesidades del presente sin comprometer la capacidad de las generaciones futuras (WCED, 1987). Esta definición crea una conexión entre la equidad social, la productividad económica y la calidad medioambiental (Bastas y Liyanage, 2019). De esta forma, el concepto de desarrollo sostenible ha captado la atención de industrias privadas, gobiernos, organizaciones no gubernamentales, académicos, ciudadanos y otras partes interesadas para resolver los desafíos de la sostenibilidad. No obstante, los problemas sociales, económicos y ambientales del desarrollo global han creado condiciones como la desigualdad, la pobreza, el cambio climático, el desarrollo tecnológico, el crecimiento económico, el sobreconsumo, entre otros. Estas condiciones impactan en la sociedad como parte de un ecosistema amplio y complejo (Hrubec, 2019). En este sentido, la definición de desarrollo sostenible proporcionada por la WCED podría ser considerada como un ideal utópico que la sociedad quiere alcanzar.

Respecto al análisis de los aspectos que involucran el desarrollo sostenible, este se conforma por tres dimensiones: económica, ambiental y social (WCED, 1987). Estas dimensiones deben ser estudiadas en conjunto para llegar a un modelo que permita una mejor comprensión para tomar decisiones y actuar sobre las complejas situaciones a las que se enfrenta el mundo actual (Waas et al., 2011). La dimensión económica se refiere al crecimiento económico como motor de creación de bienestar a largo plazo para satisfacer las necesidades esenciales, donde los intereses económicos definen el marco para la toma de decisiones, el flujo de capital financiero y la facilitación del comercio (Méndez-Picazo et al., 2021). La dimensión ambiental reconoce la necesidad de proteger, conservar y mejorar los recursos de la Tierra, así como la diversidad e interdependencia de los sistemas vivos, los bienes y servicios producidos por los ecosistemas del mundo (Han et al., 2018). Finalmente, la dimensión social se refiere a las interacciones entre las instituciones y las personas que expresan valores humanos como la igualdad de acceso a los recursos naturales, cuestiones éticas, el bienestar y la toma de decisiones colectivas (Quiroz-Niño y Murga-Menoyo, 2017).

El desarrollo sostenible es actualmente un concepto relevante en la agenda global donde se han alcanzado algunos resultados importantes (Bastas y Liyanage, 2019). Sin embargo, es imperativo que este ideal conduzca a una implementación efectiva como una estrategia de toma de decisiones en los escenarios de las partes interesadas, utilizando métricas adecuadas o herramientas cuantificables que puedan cambiar la percepción de la sostenibilidad como un concepto retórico u holístico (Sanahuja y Tezanos, 2017). Aunque existen algunos esfuerzos, es evidente que el desarrollo económico e industrial actualmente no ha alcanzado los niveles de sostenibilidad esperados. De esta manera, el desarrollo sostenible intenta trabajar sobre los retos económicos, ambientales y sociales, pero más allá de estas dimensiones, tiene otros aspectos que se deben profundizar, como el liderazgo, que, al transformarlo, el modelo sostenible será más compatible con la sociedad para impulsar acciones, estrategias y políticas reales (Newman-Storen, 2014).

El desarrollo sostenible es un concepto con grandes expectativas en la actual agenda global del cambio. Sin embargo, hay ciertos errores que cometen algunos actores que amenazan la consecución de los objetivos de sostenibilidad (Álvarez, 2016). Entre los obstáculos más comunes al adoptar el desarrollo sostenible se encuentran: el argumento de que las dimensiones de la sostenibilidad no pueden tratarse como equivalentes y la interpretación errónea del desarrollo sostenible como un concepto retórico sin solución alcanzable (Sanahuja y Tezanos, 2017). En este contexto, el espíritu emprendedor cobra mayor relevancia, ya que se caracteriza por el reconocimiento de oportunidades, el compromiso con una oportunidad, el control de los recursos, la gestión de la red de recursos y de la forma de recompensar a los participantes (Delgado et al., 2008). En el concepto de espíritu emprendedor, a la persona que ejecuta las ideas y materializa las oportunidades se le conoce como emprendedor, que es quien inicia un proceso de creación de riqueza y de adición de valor mediante una incubación de ideas, la reunión de recursos y la ejecución de acciones (Lecuna et al., 2016). El estudio del espíritu emprendedor es fundamentalmente un fenómeno basado en la acción que implica un conjunto altamente interrelacionado de procesos creativos, estratégicos y organizativos (Moroz \& Hindle, 2012). El espíritu emprendedor tiene cuatro dimensiones centrales subyacentes: la innovación, asunción de riesgos, proactividad y creatividad (Lecuna et al., 2016). Estos aspectos dan lugar a un proceso emprendedor que posteriormente se convierte en una creación tangible (Chavez et al., 2020). El proceso 
emprendedor implica todas las funciones, actividades y acciones que se asocian con la percepción de oportunidades y la creación de organizaciones para que puedan ser perseguidas.

Los emprendimientos comienzan con un evento desarticulado, discontinuo, no lineal (y generalmente único) que no puede ser estudiado con los métodos que se han desarrollado para evaluar procesos continuos y lineales (y a menudo repetibles). El emprendimiento tiene diversos modelos que los investigadores han propuesto a lo largo del tiempo para contextualizar el concepto y estudiar su impacto en los tiempos actuales (Altinay y Wang, 2011). Este es el caso del estilo de emprendimiento social que tiene como objetivo la creación de valor social, en lugar de la riqueza personal y de los accionistas. Las actividades de emprendimiento social se caracterizan por la innovación, o la creación de algo nuevo que va más allá de la simple réplica de empresas o prácticas existentes (Uribe-Toril et al., 2019). En la actualidad, las bases del emprendimiento se vinculan con los diferentes estilos de liderazgo, aportando a los ecosistemas empresariales y al desarrollo nuevas oportunidades de emprendimientos desafiantes (Barragán y Ayaviri, 2017).

En cuanto a los emprendedores, estos tienen una gran variedad de atributos, que incluyen ser visionarios, tener pasión por su idea, asumir riesgos, tener perseverancia, crear un equipo, reconocer las oportunidades y las necesidades y resolver problemas (Valenzuela-Keller et al, 2021). Según Reimers-Hild y Kinh (2009) el objetivo fundamental de los emprendedores es crear una atmósfera de innovación. La generación actual de personas se enfrenta a varios desafíos en las dimensiones social, económica y ambiental, y al igual que la transformación del desarrollo sostenible, el espíritu emprendedor también tiene una transformación en este modelo con el objetivo de vincular estos conceptos para definir, caracterizar e implementar en esta sociedad un modelo eficaz que pueda resolver los desafíos de la sostenibilidad (Ruiz-Ruano y Puga, 2016). Recientes estudios ofrecen una mejor definición del emprendimiento enfocado al desarrollo sostenible en el que estos emprendedores sostenibles son actores que quieren conciliar el crecimiento económico y contribuir a la sostenibilidad en su modelo de negocio (Naguit, 2018).

En este sentido, los emprendimientos sostenibles no se limitan a estar influenciados por los modelos estándar, sino que son capaces de transformar estas estructuras y renovarlas en función del contexto en un nuevo camino hacia un objetivo de desarrollo sostenible (Belz y Binder, 2017). La transición de la estructura estándar del espíritu emprendedor a una estructura sostenible requiere del emprendimiento sostenible: (1) un conocimiento de las necesidades, políticas y normativas en un contexto actual; (2) estar en red en múltiples partes interesadas liderando una cooperación; (3) la acción, pero teniendo en cuenta las dimensiones de la sostenibilidad; y (4) la creación de valor a través de la innovación, la creatividad y desafiando lo ya establecido (Schaltegger et al., 2016). De esta forma, cuando un emprendimiento desarrolla un ecosistema de acción, independientemente de su estructura, necesita buscar y explotar oportunidades tanto dentro de los límites del mercado como fuera de los mismos. Sin embargo, si el emprendimiento busca alcanzar un objetivo de sostenibilidad, su estructura tiene que transformarse en función del contexto.

Un punto crítico en la formación de una empresa es la supervivencia. Para el emprendimiento sostenible, la supervivencia puede verse en dos contextos diferentes: la supervivencia como miembro valorado del ecosistema y la supervivencia como preocupación continua u organización autónoma (Strotmann, 2007). Para garantizar el primer tipo de supervivencia, los emprendimientos sostenibles tendrán que identificar y perseguir las oportunidades dentro de los límites del ecosistema (oportunidades internas), ya que esto permite a la nueva empresa ampliar las ofertas actuales relacionadas con la plataforma de innovación y consolidar su posición dentro del ecosistema. Para asegurar el segundo tipo de supervivencia, el emprendimiento sostenible necesita reconocer oportunidades fuera del ecosistema, ya que esto le permite a la empresa ampliar las ofertas que ofrece al mercado.

El aprendizaje de experiencias empresariales hace que los emprendimientos evalúen las oportunidades con mayor rigor, incluidas las oportunidades percibidas para el emprendimiento sostenible (Valenzuela-Keller et al, 2021). De esta manera, el camino del emprendimiento es desafiante y requiere la creación de innovación a través de la creatividad para competir contra este complejo sistema. La innovación se produce a través de una interacción dinámica entre las partes interesadas en torno al emprendimiento (Naguit, 2018). Por tanto, el emprendimiento sostenible es un concepto que vincula el emprendimiento con la trinidad de lo económico, social y ambiental, utilizando la innovación, capacidad de resolver problemas, perseverancia, así como otras características que permiten el buen funcionamiento del modelo de emprendimiento sostenible.

En este sentido, el objetivo del presente estudio es caracterizar el emprendimiento sostenible a partir de un diseño de caso múltiple, planteando las siguientes proposiciones: Proposición 1: El desarrollo sostenible como un concepto y modelo permite poner en marcha un negocio centrado en el impacto social, económico y ambiental. Proposición 2: El reto del emprendimiento sostenible se da interna y externamente. Los emprendimientos tienen retos internos como la gestión del tiempo o de los recursos para llevar a cabo con éxito cualquier proyecto o actividad clave. Además, las políticas y regulaciones externas del gobierno también presentan desafíos para acelerar y ampliar la red de los emprendimientos sostenibles. La burocracia puede 
afectar la cooperación entre los emprendimientos y las instituciones. Proposición 3: El emprendimiento sostenible permite la formación de empresas más estables. Estas empresas pueden tardar más tiempo en alcanzar el punto de equilibrio debido a que buscan primero construir un emprendimiento con un sentido más profundo del desarrollo social, la conciencia medioambiental y el crecimiento económico.

\section{METODOLOGÍA}

El presente estudio empleó una metodología de caso múltiple donde se utilizó una entrevista estructurada para recoger la información cualitativa de emprendedores. El estudio de caso múltiple proporciona una gran cantidad de información que, tras el análisis dentro de los casos y entre ellos, revela una serie de puntos comunes y una diversidad limitada. El uso de esta metodología permite maximizar la profundidad de la información y aumenta la transferibilidad de los resultados para permitir el desarrollo de una narrativa conceptual sobre el emprendimiento sostenible. El uso de un estudio de caso múltiple ayuda a lograr una comprensión más profunda de los emprendimientos como una unidad, a través de la comparación de las similitudes y diferencias de los casos individuales seleccionados Los resultados que se obtienen de los estudios de caso múltiple suelen ser más sólidas y fiables que aquellos estudios de caso único, debido a que los estudios de caso múltiple permiten una exploración más exhaustiva de las proposiciones de la investigación y el desarrollo de la teoría (Yin, 2014).

La entrevista estuvo conformada por 11 preguntas destinadas a recabar información para comprender diversos aspectos relacionados al desarrollo sostenible. Para lograr este objetivo, fueron seleccionados cinco proyectos de emprendimiento (Tabla 1) considerando los siguientes criterios: (1) los emprendimientos seleccionados son gestionados por emprendedores que están liderando un cambio en el mercado; (2) los emprendimientos son colombianos y están ubicados en zonas rurales o urbanas; (3) los emprendimientos tienen como característica principal la creatividad, innovan, experimentan o desarrollan productos, procesos, servicios o modelos de gestión; y (4) contribuyen al desarrollo sostenible creando valor económico, impacto social y ambiental.

Tabla 1: Descripción de los emprendedores entrevistados y casos estudiados.

\begin{tabular}{|c|l|l|l|l|l|}
\hline Caso & $\begin{array}{c}\text { Nombre de la } \\
\text { empresa }\end{array}$ & $\begin{array}{c}\text { Fase en el } \\
\text { ciclo de vida }\end{array}$ & Localización & $\begin{array}{l}\text { Producto o servicio que aporta una } \\
\text { sostenibilidad en el mercado }\end{array}$ & $\begin{array}{c}\text { Líder a cargo de la } \\
\text { iniciativa emprendedora }\end{array}$ \\
\hline 1 & Organizmo & Crecimiento & $\begin{array}{l}\text { Tenjo, } \\
\text { Colombia }\end{array}$ & $\begin{array}{l}\text { Diseño, construcción y formación en } \\
\text { medios sostenibles. }\end{array}$ & Ana Gutiérrez \\
\hline 2 & Mano Cambiada & Crecimiento & $\begin{array}{l}\text { Nuqui, } \\
\text { Colombia }\end{array}$ & $\begin{array}{l}\text { Turismo sostenible y comunitario, } \\
\text { pesca sostenible. }\end{array}$ & Josefina Klinger \\
\hline 3 & $\begin{array}{l}\text { Bourbon Coffee } \\
\text { Roasters }\end{array}$ & Introducción & $\begin{array}{l}\text { Bogotá, } \\
\text { Colombia }\end{array}$ & $\begin{array}{l}\text { Comercio de cafés especiales y } \\
\text { apoyo a los caficultores locales. }\end{array}$ & $\begin{array}{l}\text { José Rosero y Paola } \\
\text { Laguna }\end{array}$ \\
\hline 4 & WOMA S.A.S & Madurez & $\begin{array}{l}\text { Medellín, } \\
\text { Colombia }\end{array}$ & $\begin{array}{l}\text { Diseño e ingeniería en prototipos de } \\
\text { tecnología textil. }\end{array}$ & Margarita Baena \\
\hline 5 & $\begin{array}{l}\text { Café Mesa de } \\
\text { los Santos }\end{array}$ & Madurez & $\begin{array}{l}\text { Los Santos, } \\
\text { Colombia }\end{array}$ & $\begin{array}{l}\text { Comercio internacional de café } \\
\text { ecológico. }\end{array}$ & Oswaldo Acevedo \\
\hline
\end{tabular}

Según Díaz-Bravo et al. (2013) una ventaja de la entrevista estructurada es la clasificación y análisis de las respuestas. Los autores señalan cuatro fases que una entrevista estructurada debe seguir: (1) preparación; (2) apertura; (3) desarrollo; y (4) cierre. En esta investigación, se utilizaron preguntas abiertas debido al tamaño de la muestra y la naturaleza de cada pregunta. Estas preguntas fueron revisadas y validadas por dos expertos en temas de emprendimiento y sostenibilidad, confirmando la relevancia, representatividad y claridad de los ítems de la entrevista. Por otro lado, para la construcción de la entrevista, se diseñó un modelo específico con la finalidad de formular preguntas relevantes para obtener conclusiones sólidas y alineadas con el objetivo y alcance del estudio (Fig. 1), siguiendo las recomendaciones de Díaz-Bravo et al. (2013). El tiempo estimado para la revisión de cada emprendimiento presencialmente fue entre uno y dos días, dependiendo del tamaño de la empresa. Esto permitió a la vez emplear una fuente externa de evidencia que brinda apoyo a la validez de la entrevista. Asimismo, el tiempo empleado para las entrevistas fue entre 45 y 60 minutos. Durante la entrevista, y para la formulación de las preguntas, se emplearon técnicas originadas en el método de pensamiento de diseño, lo que permitió que el entrevistado se sintiera cómodo durante la entrevista y respondiera a las preguntas, aportando información y conocimientos importantes sobre el emprendimiento sostenible.

La entrevista fue desarrollada con base en la revisión de literatura y guiada por el modelo presentado en la Figura1, generando las siguientes preguntas: (1) ¿Cuánto tiempo lleva realizando su trabajo?; (2) ¿Por qué realiza su trabajo actual?; (3) ¿Cuál fue la inspiración para realizar su trabajo actual?; (4) ¿Cuántos empleados tenía cuando empezó y cuántos tiene ahora?; (5) ¿Qué le motivó a incursionar en un negocio como el que tiene actualmente?; (6) ¿Ha tenido o tiene un modelo de inspiración?; (7) ¿Cuál ha sido su mayor error empresarial?; (8) ¿Por qué le apasiona su trabajo actual?; (9) ¿Ha pensado hacer algo diferente a lo que se dedica actualmente?; (10) ¿Qué opina de las políticas gubernamentales actuales?; y (11) ¿Qué es para usted la sostenibilidad o el desarrollo sostenible? 


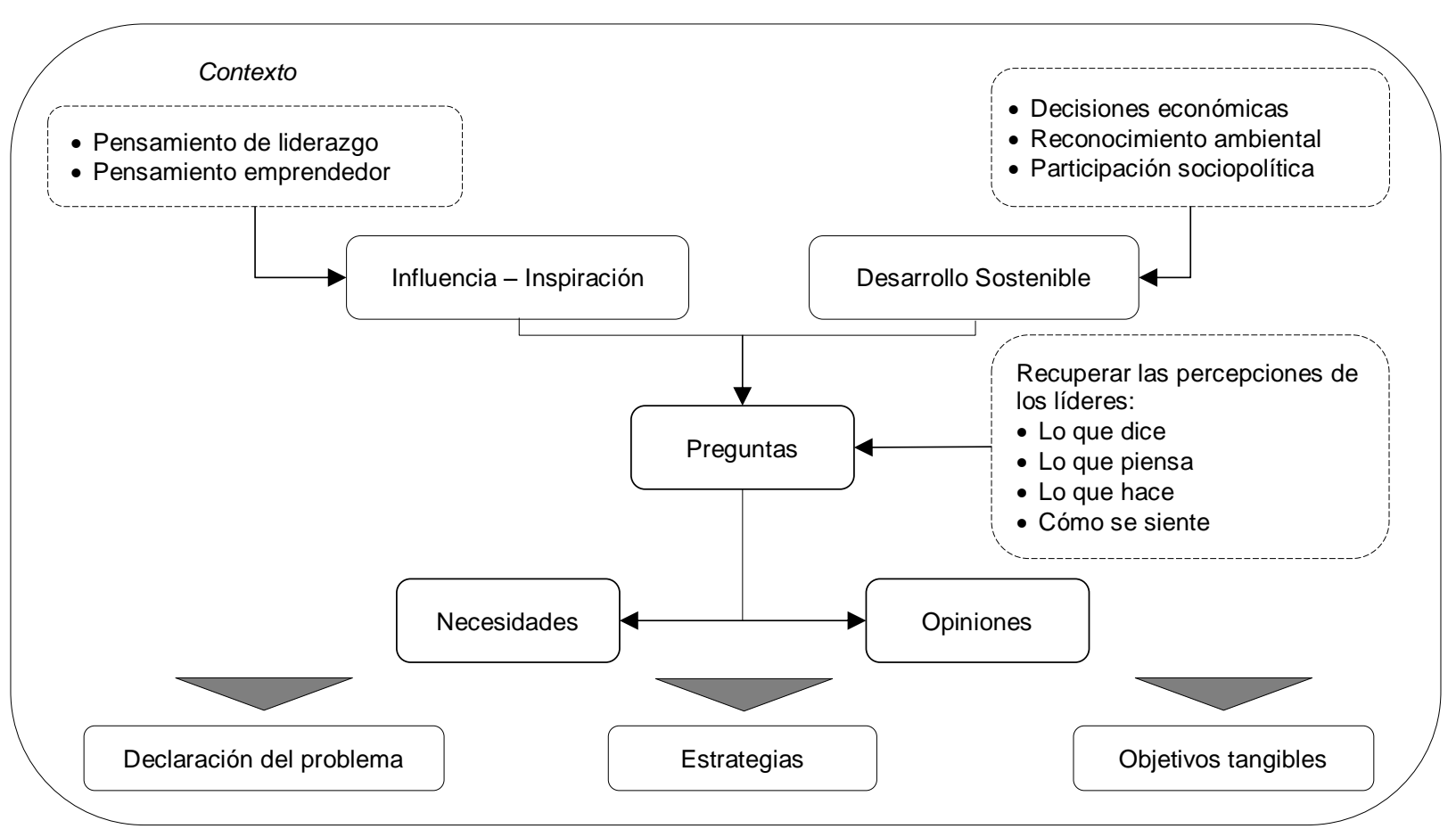

Fig. 1: Modelo para la elaboración de la entrevista sobre emprendimiento sostenible.

Una vez concluidas las entrevistas con los emprendedores, la información recogida fue procesada y analizada en concordancia con el marco teórico desarrollado y considerando lo planteado por Díaz-Bravo et al. (2013) que sugiere que los entrevistadores deben involucrarse objetivamente con los resultados obtenidos para realizar el proceso de categorización y brindar clasificaciones significativas. Las respuestas de los entrevistados se analizaron a partir de un conjunto de pasos que inició con la codificación de las respuestas para luego categorizarlas en temáticas comunes y finalmente establecer conclusiones que permiten una explicación general agrupadas en los temas comunes. Asimismo, todos los datos fueron procesados manualmente debido al tamaño de la muestra y al número de preguntas establecidas en la entrevista.

\section{RESULTADOS}

Las entrevistas realizadas a los líderes involucrados en emprendimientos sostenibles, expusieron los factores, estrategias y percepciones de la sostenibilidad como concepto, modelo de desarrollo para iniciar un emprendimiento, roles que desempeñan para un liderazgo efectivo en función del cumplimiento de los objetivos de la empresa y cómo diseñaron acciones estratégicas en áreas centrales de la gestión hacia el desarrollo de un emprendimiento sostenible exitoso (Tabla 2). Los resultados indican que la influencia de los emprendedores está acompañada de una inspiración que les permitió convertirse en líderes, y más aún, en emprendedores sostenibles. En este sentido, los emprendedores indicaron que su mayor inspiración para convertirse en líderes y decidir emprender fueron las condiciones de Colombia respecto a la educación, territorio, innovación, desigualdad y desarrollo social. Sin embargo, concibieron estas nociones como dificultades en un primer momento, pero tomaron esos retos con una visión diferente y los convirtieron en oportunidades de cambio. Además, los entrevistados coincidieron en que, al convertirse en directivos, se vieron influidos por un modelo inspirador que les ayudó a tomar el rumbo de una gestión de éxito centrada en la sostenibilidad. Estos modelos inspiradores provenían de trabajos previos, voluntariados y de la creación de redes con otros líderes. En este último aspecto, la creación de redes entre empresarios o la buena relación entre empleado y empleador, les permitió fortalecer sus habilidades de emprendimiento con base en sus actividades y su estilo de vida.

Los emprendedores iniciaron sus negocios en la misma línea de experiencia en la que habían trabajado, argumentando que tenían pasión por aquello para lo que se habían preparado como profesionales. Además, porque las oportunidades que identificaron tenían retos urgentes que atender en el corto plazo más que generar ganancias. En este escenario, los emprendedores entendieron desde el inicio la razón para hacerlo, lo que les proporciona otra perspectiva de gestión como priorizar el desarrollo social, la construcción del entorno, el desarrollo de la innovación y la educación. Por otro lado, de las cinco empresas, dos comenzaron con dos empleados, otra empresa también comenzó con dos empleados y una empresa comenzó con nueve empleados (todos ellos totalmente comprometidos con el emprendimiento y los objetivos del emprendedor). A partir esto, se establece que la relación entre los empleados y los emprendedores fue amistosa, profesional y efectiva, fortalecida por las acciones del emprendedor, que transformaron y empoderaron a sus empleados para lograr los objetivos de la empresa, creando una pasión común para el crecimiento de la empresa. 
Tabla 2: Resultados obtenidos y comparación con las proposiciones.

\begin{tabular}{|c|c|c|c|c|c|}
\hline \multirow[b]{2}{*}{$\begin{array}{l}\text { Nombre } \\
\text { de la } \\
\text { empresa }\end{array}$} & \multirow[b]{2}{*}{ Industria } & \multirow[b]{2}{*}{$\begin{array}{c}\text { Descripción y objetivos } \\
\text { alcanzados }\end{array}$} & \multicolumn{3}{|c|}{ Respuesta a las proposiciones } \\
\hline & & & $\begin{array}{c}\text { Comprensión del } \\
\text { desarrollo sostenible } \\
\text { como un concepto y } \\
\text { modelo }\end{array}$ & $\begin{array}{c}\text { Reto del emprendimiento } \\
\text { sostenible }\end{array}$ & $\begin{array}{c}\text { El emprendimiento } \\
\text { sostenible en empresas } \\
\text { estables }\end{array}$ \\
\hline Organizmo & $\begin{array}{l}\text { Diseño, } \\
\text { educación y } \\
\text { construcción }\end{array}$ & $\begin{array}{l}\text { - Colaboración de } \\
\text { programas educativos con } \\
\text { universidades y } \\
\text { académicos nacionales e } \\
\text { internacionales con el fin } \\
\text { de transferir conocimientos } \\
\text { relacionados con la } \\
\text { bioconstrucción utilizando } \\
\text { diversos materiales } \\
\text { orgánicos. } \\
\text { - Desarrollo de } \\
\text { infraestructuras } \\
\text { comunitarias en zonas } \\
\text { rurales de Colombia, } \\
\text { beneficiando el bienestar y } \\
\text { la concienciación de la } \\
\text { gestión de residuos de la } \\
\text { población. } \\
\text { - Desarrollo de productos } \\
\text { sostenibles para empresas } \\
\text { privadas de arquitectura y } \\
\text { construcción. }\end{array}$ & $\begin{array}{l}\text { Es la integración con la } \\
\text { comunidad, el } \\
\text { seguimiento de la } \\
\text { infraestructura social y } \\
\text { ambiental, en conjunto, } \\
\text { para gestionar los } \\
\text { recursos disponibles y } \\
\text { lograr el crecimiento } \\
\text { local. }\end{array}$ & $\begin{array}{l}\text { - Buenas relaciones con } \\
\text { los empleados, } \\
\text { proporcionándoles } \\
\text { espacios de } \\
\text { colaboración y } \\
\text { retroalimentación para } \\
\text { fortalecer la empresa. } \\
\text { - Capacitación a los } \\
\text { empleados para } \\
\text { promover y crear } \\
\text { proyectos con un ideal } \\
\text { de sostenibilidad. }\end{array}$ & $\begin{array}{l}\text { Crear una red de } \\
\text { contactos para el } \\
\text { intercambio de ideas } \\
\text { entre todas las partes } \\
\text { interesadas. }\end{array}$ \\
\hline $\begin{array}{l}\text { Mano } \\
\text { Cambiada }\end{array}$ & Turismo & $\begin{array}{l}\text { - Desarrollo cultural de las } \\
\text { comunidades del Pacífico } \\
\text { para dotarlas de un sentido } \\
\text { de pertenencia a sus } \\
\text { orígenes, complementado } \\
\text { con educación, para que } \\
\text { se conviertan en } \\
\text { empresarios locales } \\
\text { aprovechando sus } \\
\text { recursos naturales. } \\
\text { - Experiencia educativa y de } \\
\text { creación de redes entre los } \\
\text { nativos y la población } \\
\text { urbana o los turistas. } \\
\text { - Cuidado del Parque } \\
\text { Nacional Natural Utría y el } \\
\text { desarrollo de programas } \\
\text { educativos para los niños } \\
\text { locales, principalmente en } \\
\text { Nuqui. }\end{array}$ & $\begin{array}{l}\text { Es el equilibrio para } \\
\text { encontrar el sentido de la } \\
\text { vida, generando sentido } \\
\text { individual y colectivo a } \\
\text { través de un proceso de } \\
\text { autodescubrimiento y } \\
\text { empoderamiento en las } \\
\text { comunidades locales, con } \\
\text { el fin de resolver retos } \\
\text { específicos sin } \\
\text { comprometer los } \\
\text { recursos naturales. }\end{array}$ & $\begin{array}{l}\text { - La participación social } \\
\text { consiste en la creación } \\
\text { de talleres y eventos } \\
\text { sociales, promoviendo el } \\
\text { desarrollo entre la } \\
\text { población local. } \\
\text { - Empoderamiento de la } \\
\text { población local a partir } \\
\text { del ideal de "minga", que } \\
\text { significa un intercambio } \\
\text { justo de conocimientos, } \\
\text { bienes y servicios en } \\
\text { beneficio del desarrollo } \\
\text { social más que de los } \\
\text { ingresos económicos. }\end{array}$ & $\begin{array}{l}\text { - El desarrollo } \\
\text { comunitario coincide } \\
\text { de manera profunda } \\
\text { integrando la } \\
\text { participación y el } \\
\text { desarrollo de } \\
\text { productos. } \\
\text { - Creación de redes de } \\
\text { trabajo. }\end{array}$ \\
\hline $\begin{array}{l}\text { Bourbon } \\
\text { Coffee } \\
\text { Roasters }\end{array}$ & $\begin{array}{l}\text { Industria del } \\
\text { café }\end{array}$ & $\begin{array}{l}\text { - Dos tiendas de café } \\
\text { situadas en Bogotá. } \\
\text { - Todo el café que venden } \\
\text { procede de pequeñas } \\
\text { explotaciones cafeteras } \\
\text { orgánicas de Colombia, } \\
\text { creando una auténtica y } \\
\text { sólida conexión entre el } \\
\text { campo y la ciudad. } \\
\text { - El precio del café se paga } \\
\text { en función de los costes de } \\
\text { producción de la } \\
\text { explotación (no se basa en } \\
\text { el precio de mercado). }\end{array}$ & $\begin{array}{l}\text { Es una cadena en la que } \\
\text { cada proceso del } \\
\text { proyecto tiene que ser } \\
\text { transparente, eficiente y } \\
\text { sin comprometer el } \\
\text { desperdicio de recursos } \\
\text { locales. Después de esto, } \\
\text { cada actor involucrado y } \\
\text { siguiendo prácticas } \\
\text { adecuadas debe obtener } \\
\text { no sólo un beneficio } \\
\text { económico sino personal, } \\
\text { logrando así un éxito en } \\
\text { la vida. }\end{array}$ & $\begin{array}{l}\text { - Potenciación de los } \\
\text { proveedores, } \\
\text { optimizando sus } \\
\text { explotaciones para } \\
\text { producir un café de } \\
\text { mejor calidad mediante } \\
\text { el asesoramiento técnico } \\
\text { y el pago de precios } \\
\text { equitativos. } \\
\text { - Creación de valor en la } \\
\text { cadena siendo abierta a } \\
\text { través de las prácticas } \\
\text { con los clientes, } \\
\text { incentivándolos a } \\
\text { entender el café como } \\
\text { una cultura de desarrollo } \\
\text { para el beneficio común } \\
\text { en Colombia. }\end{array}$ & $\begin{array}{l}\text { - Cree en la } \\
\text { transparencia para las } \\
\text { partes interesadas, } \\
\text { incluidos los clientes } \\
\text { que aportan la } \\
\text { información sobre } \\
\text { prácticas, procesos y } \\
\text { actividades. Esto } \\
\text { refuerza el trabajo en } \\
\text { red. } \\
\text { - Implicación de los } \\
\text { empleados en la } \\
\text { generación de ideas } \\
\text { para la convergencia, } \\
\text { expansión y mejora de } \\
\text { la presencia de la } \\
\text { empresa en el } \\
\text { mercado. }\end{array}$ \\
\hline
\end{tabular}


Tabla 2: continuación

\begin{tabular}{|c|c|c|c|c|c|}
\hline & & & \multicolumn{3}{|c|}{ Respuesta a las proposiciones } \\
\hline $\begin{array}{l}\text { Nombre } \\
\text { de la } \\
\text { empresa }\end{array}$ & Industria & $\begin{array}{l}\text { Descripción y objetivos } \\
\text { alcanzados }\end{array}$ & $\begin{array}{l}\text { Comprensión del } \\
\text { desarrollo sostenible } \\
\text { como un concepto y } \\
\text { modelo }\end{array}$ & $\begin{array}{l}\text { Reto del emprendimiento } \\
\text { sostenible }\end{array}$ & $\begin{array}{l}\text { El emprendimiento } \\
\text { sostenible en empresas } \\
\text { estables }\end{array}$ \\
\hline $\begin{array}{l}\text { WOMA } \\
\text { S.A.S. }\end{array}$ & $\begin{array}{l}\text { Innovación y } \\
\text { tecnología }\end{array}$ & $\begin{array}{l}\text { - Empresa pionera en la } \\
\text { externalización de la } \\
\text { innovación y la I+D en } \\
\text { Colombia. } \\
\text { - Sus soluciones se centran } \\
\text { en proporcionar } \\
\text { accesibilidad, educación, } \\
\text { economía y no } \\
\text { contaminación del medio } \\
\text { ambiente. } \\
\text { - Impulsa la educación de } \\
\text { los jóvenes para que } \\
\text { innoven en beneficio de la } \\
\text { comunidad local, } \\
\text { generalmente en Medellín. }\end{array}$ & $\begin{array}{l}\text { Es el ciclo propio y la } \\
\text { reinvención del negocio } \\
\text { en función del contexto y } \\
\text { sin comprometer la } \\
\text { sinergia establecida en } \\
\text { las operaciones } \\
\text { financieras, el desarrollo } \\
\text { social y la gestión } \\
\text { medioambiental. }\end{array}$ & $\begin{array}{l}\text { - Apoya y fomenta el } \\
\text { empleo de los jóvenes } \\
\text { por la creatividad que } \\
\text { pueden aportar a una } \\
\text { empresa. } \\
\text { - Cree en el trabajo en } \\
\text { equipo. } \\
\text { - Inspiración para ser un } \\
\text { agente de cambio a } \\
\text { través de la } \\
\text { externalización de la } \\
\text { innovación. }\end{array}$ & $\begin{array}{l}\text { - Trabaja sobre la } \\
\text { estructura organizativa } \\
\text { de un proyecto y el } \\
\text { conocimiento de las } \\
\text { redes. } \\
\text { - Desafía lo ya } \\
\text { establecido utilizando } \\
\text { la creatividad como } \\
\text { herramienta para el } \\
\text { desarrollo de } \\
\text { productos. }\end{array}$ \\
\hline $\begin{array}{l}\text { Café Mesa } \\
\text { de los } \\
\text { Santos }\end{array}$ & $\begin{array}{l}\text { Agricultura e } \\
\text { industria del } \\
\text { café }\end{array}$ & $\begin{array}{l}\text { - La mayor empresa } \\
\text { exportadora de cafés } \\
\text { especiales de Colombia, } \\
\text { con premios y certificados } \\
\text { de calidad en materia de } \\
\text { gestión de recursos, } \\
\text { reforestación y gestión } \\
\text { humana. } \\
\text { - Fuente de empleo para la } \\
\text { población local, los } \\
\text { agricultores y otros } \\
\text { proveedores locales en la } \\
\text { región de Santander. } \\
\text { - Salarios justos y } \\
\text { competitivos. } \\
\text { Certificaciones USDA y } \\
\text { JAS. }\end{array}$ & $\begin{array}{l}\text { Implica el uso de los } \\
\text { recursos naturales y } \\
\text { humanos adecuados en } \\
\text { los que se ubica un } \\
\text { proyecto para entregar } \\
\text { correctamente la } \\
\text { infraestructura que se } \\
\text { requiere y luego obtener } \\
\text { ingresos justos para todo } \\
\text { el ecosistema compartido } \\
\text { en términos tarifarios. }\end{array}$ & $\begin{array}{l}\text { - La reforestación de } 730 \\
\text { hectáreas en un suelo } \\
\text { seco creó y transmitió un } \\
\text { fuerte mensaje de } \\
\text { desarrollo social y } \\
\text { natural para la población } \\
\text { local de los alrededores } \\
\text { de Los Santos. } \\
\text { - Trabajo conjunto entre } \\
\text { las zonas rurales y las } \\
\text { urbanas (clientes } \\
\text { internacionales). } \\
\text { - Apoyo a la I+D y la } \\
\text { formación de los } \\
\text { empleados } \\
\text { permanentes. }\end{array}$ & $\begin{array}{l}\text { - Identifica las } \\
\text { oportunidades que } \\
\text { ofrece el mercado } \\
\text { para crear estrategias } \\
\text { que impacten } \\
\text { positivamente en las } \\
\text { partes interesadas. } \\
\text { - Cree en la } \\
\text { investigación y el } \\
\text { trabajo en } \\
\text { colaboración para } \\
\text { crear u optimizar los } \\
\text { procesos para ofrecer } \\
\text { un mejor producto a } \\
\text { los clientes. }\end{array}$ \\
\hline
\end{tabular}

Respecto a los retos más importantes a los que se han enfrentado a lo largo de la gestión de sus empresas sostenibles, los entrevistados indicaron que, al principio, priorizaron las dimensiones social y ambiental para alcanzar los objetivos de sostenibilidad. Sin embargo, esta decisión los llevó a luchar contra las dificultades económicas y a tomar decisiones estratégicas erróneas para mantener la empresa durante la fase inicial de operaciones. Los emprendedores coincidieron en que el desconocimiento del proceso de gestión de proyectos fue la razón principal para llegar a ese punto. Además, los líderes explicaron que las políticas gubernamentales en materia de desarrollo ambiental y social habían sido diferentes y parcialmente inexactas al momento de converger los objetivos de la empresa en materia de desarrollo social y ambiental. Debido a que las dimensiones del desarrollo sostenible tienen un impacto en un escenario específico, el gobierno se convierte en una parte interesada de cualquier emprendimiento sostenible y las políticas tienden a tener una excesiva burocracia, una estructuración más lenta de objetivos y actividades para lograr los objetivos en un modelo de desarrollo sostenible.

Por otro lado, se identificó que las empresas sostenibles tienen un ciclo de vida entre 3 y 20 años. Cabe destacar que una de ellas, la más joven, se encuentra en el mismo nivel de estabilidad operativa y financiera que las demás empresas analizadas. Este hallazgo indica que la duración de la empresa puede ser vista como una primera medida del impacto que una iniciativa empresarial sostenible tiene en las dimensiones económica, social y ambiental de un entorno específico a largo plazo. Según lo analizado y de acuerdo con el ciclo de vida de los emprendimientos, se encontró un emprendimiento en la etapa de introducción, donde la sostenibilidad forma parte de los procesos y resultados, pero no es el rasgo diferenciador identificado por los usuarios, por lo que el impacto que tiene es bajo. Dos emprendimientos se encontraron en la etapa de crecimiento, en el que la sostenibilidad es identificada por los usuarios como una parte más de la empresa, aunque aún no es un rasgo diferenciador respecto a otros emprendimientos. Finalmente, dos emprendimientos estuvieron en la fase de madurez, donde la sostenibilidad es un componente diferenciador que permite a la empresa aprovechar este valor agregado para generar un impacto positivo en el mercado, otorgándoles ventaja competitiva. 
En cuanto a la concepción de los emprendimientos sostenibles, los empresarios mencionaron diversas características vinculadas a sus influencias y a la pasión que los llevó a iniciar el negocio. Para ellos, el detonante para iniciar un emprendimiento centrado en la sostenibilidad fue la pasión por ideas claramente definidas, como la educación, el conocimiento, la innovación, el reconocimiento ambiental, la conciencia comunitaria y las preferencias personales. De esta manera, el escenario de desarrollo al que se enfrenta la sociedad tiene que incorporar líderes sostenibles en las organizaciones y en los sistemas educativos, quienes tienen un papel más importante en la toma de decisiones de riesgo y en la expansión de una red diversa. A partir de los casos analizados, se demostró la coherencia de las proposiciones planteadas en el estudio (Tabla 2).

Todos los emprendimientos evaluados se orientan hacia el desarrollo sostenible, proponiendo o adaptando modelos en este ámbito con el fin de reorientar sus estrategias, procesos y políticas hacia objetivos alcanzables y realizables para impulsar un cambio efectivo y una progresión escalable. Es importante destacar que todas estas empresas están actualmente en funcionamiento y presentan el impacto del espíritu empresarial sostenible en sus comunidades. Asimismo, la percepción de los emprendedores hacia el desarrollo sostenible se da como un componente de la orientación emprendedora al añadir valor a sus negocios, reeducando a los clientes, exportando sus conocimientos y operaciones al extranjero para ser más competitivos, así como transformando las industrias para esperar el surgimiento de nuevos emprendimientos sostenibles.

\section{DISCUSIÓN}

El objetivo del estudio fue caracterizar el emprendimiento sostenible a partir de un diseño de caso múltiple. Para ello, se presentó una aproximación a los conceptos de sostenibilidad y emprendimiento, centrando estos enfoques en el marco del desarrollo sostenible en función de las condiciones de un entorno empresarial global y competitivo. Esto puede ser un paso inicial para desafiar y cambiar los sistemas y modelos de pensamiento actuales sobre cómo se orienta un emprendedor, y cómo iniciar un proyecto teniendo el desarrollo sostenible como objetivo principal. Además, el análisis de los emprendimientos sostenibles y la gestión de sus emprendedores proporciona información útil para el desarrollo de un futuro modelo orientado al cumplimiento de los retos de sostenibilidad que ayude a dar un paso adelante en la construcción de proyectos sostenibles exitosos desde el núcleo de su gestión. Como señalan Campo-Ternera et a. (2018), en los últimos 10 años ha habido un mayor estudio sobre la relación entre emprendimiento, innovación, desarrollo sostenible y formación para el emprendimiento en Colombia. No obstante, según los autores plantean la necesidad de formular estrategias frente al desarrollo sostenible a partir de un comportamiento emprendedor e innovador, donde la educación es el principal medio para enfrentar los cambios y las necesidades de la sociedad en un entorno dinámico y competitivo.

Las características encontradas a partir de los hallazgos obtenidos, pueden resumirse en las siguientes: (1) contexto del sistema como la competencia en la que un emprendedor tiene que investigar, analizar y entregar estrategias de acuerdo a las condiciones macro y microeconómicas donde se ubica el proyecto; (2) valor de la innovación definido como la forma en que el emprendedor entrega la innovación, pero enfatizando en los resultados que afectan a todos los actores involucrados en el emprendimiento; (3) capacidades referidas al conocimiento que tiene el emprendedor para desarrollar un proyecto exitoso; (4) gestión de la interacción definida como las redes que pueden ser creadas o mantenidas por el emprendedor en el largo plazo y que lleva a una cooperación mutua para el beneficio de los actores; (5) la colectividad definida como el trabajo entre el líder y el seguidor para aumentar el impacto en el modelo; (6) la conectividad referida al hecho de servir a los actores e inspirarlos; (7) la creatividad como herramienta impulsora de la innovación; (8) la continuidad definida como el esfuerzo por buscar y centrar el trabajo en un objetivo común; (9) el reconocimiento del contexto, en el que el líder analiza el contexto social, político y medioambiental para establecer estrategias que unifiquen a la comunidad y a otros actores; y (10) la conciencia, que es la visión del mundo, las creencias, los modelos mentales y las actitudes del líder.

La contribución del estudio sobre las empresas sostenibles aporta una serie de conocimientos teóricos y prácticos. A nivel teórico, el análisis conceptual del emprendimiento sostenible sugiere que es considerado por los líderes en el contexto actual, los ecosistemas y otros desafíos que enfrenta la sociedad. Por tanto, es vital explorar nuevos marcos que conduzcan a un desarrollo sostenible no utópico, utilizando el núcleo de la sostenibilidad y redefiniendo este modelo en función del contexto actual, considerando las necesidades que tiene la sociedad. A nivel práctico, en cuanto a la visión de cómo los emprendedores desarrollan empresas basadas en el concepto de sostenibilidad, se puede concluir que aplican un estilo de liderazgo sostenible y que ponen más esfuerzo en la ejecución de los procesos o tareas que en el diseño o planificación de los mismos, sobre todo porque estos líderes creen en el trabajo en red y colaborativo que se establece entre los diferentes actores que persiguen un mismo objetivo. Desde esta perspectiva, los casos consultados ponen de manifiesto que, el emprendimiento sostenible en el sector de las pymes sigue siendo incipiente, siguiendo lo señalado por Hitchens et al. (2003), de que el rendimiento medioambiental es una limitación necesaria para las actividades de la empresa y una interrupción de las actividades relacionadas con la producción. 
Al aplicar los conceptos de sostenibilidad y emprendimiento en la creación de un nuevo negocio, el emprendedor tomará el desarrollo sostenible como un componente para la orientación emprendedora, a partir de que el emprendedor integró el conocimiento empresarial sobre el emprendimiento convencional con el conocimiento de las cuestiones de sostenibilidad (Altinay y Wang, 2011). Por tanto, el rol del emprendedor en la creación de una empresa sostenible, mediante la aplicación de un marco de desarrollo sostenible, será la transformación de su proyecto empresarial en un negocio competitivo, que se enfrente a los desafíos en un contexto económico, social y ambiental internacional volátil (Naguit, 2018). Esta afirmación, confirma las proposiciones planteadas, mostrando la complejidad en la explotación del concepto de sostenibilidad hacia un desarrollo eficiente del emprendimiento.

Los conceptos e ideas centrales del presente estudio, apoyan el análisis inicial de cinco emprendimientos sostenibles en diferentes regiones de Colombia. Este estudio buscó caracterizar el emprendimiento sostenible a partir de un diseño de caso múltiple en relación con la comprensión del desarrollo sostenible como concepto y modelo, los retos del emprendimiento sostenible, y la capacidad del emprendimiento sostenible para la formación de empresas estables. Asimismo, este trabajo brinda una aproximación para conectar y profundizar en las competencias emprendedoras para lograr los objetivos alcanzables que circunscribe este concepto. Además, busca ser un marco funcional que explique el proceso que impulsa a determinados gestores a la concepción de emprendimientos con un componente sostenible, convirtiéndolos en proyectos emprendedores exitosamente sostenibles.

\section{CONCLUSIONES}

Desde el análisis conceptual hasta el trabajo de campo realizado, este estudio concluye que el papel de los emprendedores en la actualidad tiene un efecto vital en la aplicación del concepto de desarrollo sostenible, ya que, al presentarse como un conocimiento, un proyecto o un emprendimiento, las competencias del emprendedor pueden llevar los desafíos e ideas de la sostenibilidad a acciones concretas que impacten en los actores y en el contexto en el que se desenvuelven.

\section{REFERENCIAS}

Altinay, L., y Wang, C.L., The influence of an entrepreneur's socio-cultural characteristics on the entrepreneurial orientation of small firms, https://doi.org/10.1108/14626001111179749, J. Small Bus. Enterp. Dev., 18(4), 673-694 (2011)

Álvarez, A.M., Retos de América Latina: agenda para el desarrollo sostenible y negociaciones del siglo XXI, https://doi.org/10.1016/j.rpd.2016.08.002, Probl. Desarro., 47(186), (2016)

Barragán, M.C., y Ayaviri, V.D., Innovación y emprendimiento, y su relación con el desarrollo local del pueblo de Salinas de Guaranda, Provincia Bolívar, Ecuador, https://doi.org/10.4067/S0718-07642017000600009, Inf. Tecnol., 28(6), 71-80 (2017)

Bastas, A., y Liyanage, K., Setting a framework for organisational sustainable development, https://doi.org/10.1016/j.spc.2019.06.005, Sustain. Prod. Consum., 20, 207-229 (2019)

Belz, F.M., y Binder, J.K., Sustainable entrepreneurship: a convergent process model, https://doi.org/10.1002/bse.1887, Bus. Strat. Env., 26(1), 1-17 (2017)

Campo-Ternera, L., Amar-Sepúlveda, P., Olivero, E., y Huguett, S., Emprendimiento e innovación como motor de desarrollo sostenible: estudio bibliométrico (2006-2016), Rev. Ciencias Soc., 24(4), 26-37 (2018)

Chavez, R., Yu, W., y otros tres autores, Can entrepreneurial orientation improve sustainable development through leveraging internal lean practices?, https://doi.org/10.1002/bse.2496, Bus. Strateg. Environ., 29(6), 2211-2225 (2020)

Delgado, M.A., Gómez, L., Martínez, A.M., y Vázquez, E., Determinantes sociales y cognitivos en el espíritu emprendedor: un estudio exploratorio entre estudiantes argentinos, Cuad. Gest., 8(1), 11-24 (2008)

Díaz-Bravo, L., Torruco-García, U., Martínez-Hernández, M., y Varela-Ruiz, M., La entrevista, recurso flexible y dinámico, Investig. en Educ. Médica, 2(7), 162-167 (2013)

Han, H., Lee, M.J., y Kim, W., Antecedents of green loyalty in the cruise industry: sustainable development and environmental management, https://doi.org/10.1002/bse.2001, Bus. Strat. Env., 27(3), 323-335 (2018)

Hitchens, D., Clausen, J., y otros tres autores, Competitiveness, environmental performance and management of SMEs, Greener Manag. Int., 44, 45-57 (2003)

Hrubec, M., Social and environmental conflicts or sustainable development? Positive alarmism, https://doi.org/10.15448/1984-7289.2019.2.33782, Civ. - Rev. Ciências Sociais, 19(2), 281-295 (2019)

Lecuna, A., Cohen, B., y Chavez, R., Characteristics of high-growth entrepreneurs in Latin America, https://doi.org/10.1007/s11365-016-0402-y, Int. Entrep. Manag. J., 13(1), 141-159 (2017) 
Méndez-Picazo, M.-T., Galindo-Martín, M.-A., y Castaño-Martínez, M.-S., Effects of sociocultural and economic factors on social entrepreneurship and sustainable development, https://doi.org/10.1016/j.jik.2020.06.001, J. Innov. Knowl., 6(2), 69-77 (2021)

Moroz, P.W., y Hindle, K., Entrepreneurship as a process: toward harmonizing multiple perspectives, https://doi.org/10.1111/j.1540-6520.2011.00452.x, Entrep. Theory Pract., 36(4), 781-818 (2012)

Naguit, M.C., Sustainable entrepreneurship: the triple bottom line and business performance approach, https://doi.org/10.18848/2325-1115/CGP/v14i03/51-64, Int. J. Soc. Sustain. Econ. Soc. Cult. Context, 14(3), 51-64 (2018)

Newman-Storen, R., Leadership in sustainability: creating an interface between creativity and leadership theory in dealing with "wicked problems", https://doi.org/10.3390/su6095955, Sustain., 6(9), 5955-5967 (2014)

Quiroz-Niño, C., y Murga-Menoyo, Social and solidarity economy, sustainable development goals, and community development: the mission of adult education and training, https://doi.org/10.3390/su9122164, Sustain., 9(12), 2164 (2017)

Reimers-Hild, C., y King, J.W., Six questions for entrepreneurial leadership and innovation in distance education, Online J. Distance Learn. Adm., 12(4), (2009)

Ruiz-Ruano, A.-M., y Puga, J.L., Sustainable entrepreneurship in universities and environmental values, https://doi.org/10.1080/21711976.2015.1114218, PsyEcology, 7(1), 1-24 (2016)

Sanahuja, J.A., y Tezanos, S., "Del milenio a la sostenibilidad": retos y perspectivas de la Agenda 2030 para el desarrollo sostenible, https://doi.org/10.5209/POSO.51926, Polit. y Soc., 54(2), 521-543 (2017)

Schaltegger, S., Lüdeke-Freund, F., y Hansen, E.G., Business models for sustainability: a co-evolutionary analysis of sustainable entrepreneurship, innovation, and transformation, https://doi.org/10.1177/1086026616633272, Organ. Environ., 29(3), 264-289 (2016)

Stromann, H., Entrepreneurial survival, https://doi.org/10.1007/s11187-005-8859-z, Small Bus. Econ., 28(1), 87-104 (2007)

Sulphey, M.M., The present and future of education for sustainable development: a fact sheet, https://doi.org/10.1504/IJEWE.2019.103393, Int. J. Environ. Work. Employ., 5(3), 220-234 (2019)

Tsaurkubule, A., Towards sustainable development: changing the model of social policy in Latvia, https://doi.org/10.9770/jssi.2016.5.4(10), J. Secur. Sustain. Issues, 5(4), 576-588 (2016)

Uribe-Toril, J., De Pablo, J., Ruiz-Real, J.L., y Ramos, J., Literatura científica sobre emprendimiento social y su impacto en el ámbito iberoamericano, https://doi.org/10.31876/rcs.v25i3.27353, Rev. Ciencias Soc., 25(3), 10-29 (2019)

Valenzuela-Keller, A.A., Gálvez-Gamboa, F.A., Contreras, D.R. y Parraguez, F.P., Análisis del perfil emprendedor para la formación de las nuevas generaciones de jóvenes chilenos, https://doi.org/10.4067/S0718-07642021000100209, Inf. Tecnol., 32(1), 209-216 (2021)

Waas, T., Hugé, J., Verbruggen, A., y Wright, T., Sustainable development: a bird's eye view, https://doi.org/10.3390/su3101637, Sustain., 3(10), 1637-1661 (2011)

WCED, World Commission on Environment and Development, Our Common Future, Oxford University Press, Oxford, Inglaterra (1987)

Yin, R.K., Case Study Research: Design and Methods, 5a ed., Sage, California, USA (2014) 